\title{
DPP-4-Hemmer
}

\section{Geringe Glukosevariabilität, wenig Nebenwirkungen}

Neben den Vorteilen seiner Wirkstoffklasse besitzt der DPP-4-Hemmer Vildagliptin substanzspezifische Eigenschaften, die zum Therapieerfolg beitragen könnten.

Gliptine setzen mit ihrer Wirkung nicht nur an den Betazellen des Pankreas an, sondern regulieren auch die bei Typ-2-Diabetes gestörte Glukagonsekretion der Alphazellen. So wird die Inselzelldysfunktion umfassend in den Behandlungsansatz einbezogen, was laut Prof. Diethelm Tschöpe, Bad Oeynhausen, eine wichtige Eigenschaft für ein Antidiabetikum ist. Auch die dauerhafte effektive $\mathrm{HbA}_{1 c}$-Senkung über bis zu 52 Wochen ist inzwischen für Vildagliptin belegt. Allerdings sagt nicht allein die $\mathrm{HbA}_{1 c}$-Senkung etwas über die Wirksamkeit eines Antidiabetikums aus, so Tschöpe. Was wirklich zählt, sei die Gesamtbalance aus Euglykämie und dem Ausbleiben schädlicher Effekte.

Ein wichtiger Aspekt für die Wirksamkeit könnte eine geringe Glukosevariabilität sein. In einer aktuellen Studie mit Vildagliptin versus Sitagliptin in Standarddosierungen fiel das Ergebnis in dieser Hinsicht zugunsten von Vildagliptin aus. Dafür könnte die unterschiedliche Molekülstruktur der beiden Substanzen ein Grund sein, erläu- terte Dr. Chantal Mathieu, Leuven (Belgien). Im Gegensatz zum kompetitiven DPP-4Hemmer Sitagliptin ist Vildagliptin kovalent an das aktive Zentrum des Enzyms gebunden, was für eine länger anhaltende Wirkung spricht. Bei den schädlichen Effekten einer antiglykämischen Therapie stehen Hypoglykämien an erster Stelle. So ist etwa aus der VADT-Studie bekannt, dass sie ein mindestens ebenso großer Prädiktor für die kardiovaskuläre Mortalität sind wie ein durchgemachter Herzinfarkt, sagte Tschöpe. Mit Vildagliptin ist die Vermeidung von Unterzuckerungen effektiv möglich.

Zusätzliche Vorteile sind neben der Gewichtsneutralität der DPP-4-Hemmer auch

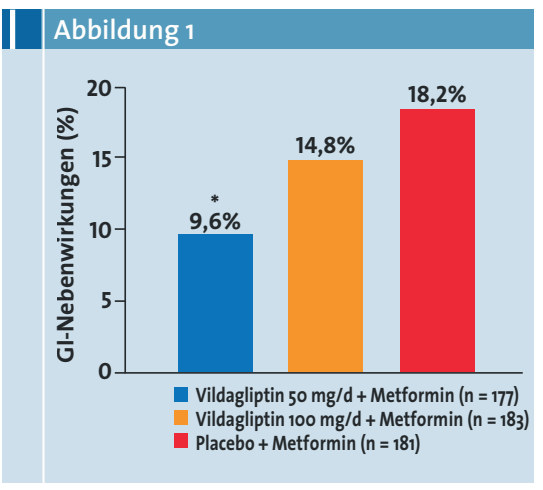

Gastrointestinale Verträglichkeit von Vildagliptin versus Placebo $\left({ }^{*} p<0,001\right)$. weitere Zeichen einer guten Verträglichkeit. So habe Vildagliptin (Galvus ${ }^{\circledR}$ ) bislang keine Hinweise für eine erhöhte Inzidenz von kardiovaskulären, hepatischen oder Pankreatitiserkrankungen geliefert. In Kombination mit dem First-line-Therapeutikum Metformin (Eucreas ${ }^{\circledR}$ ) hat sich zudem ein weiterer wichtiger Aspekt offenbart, auf den auch Prof. Emanuele Bosi, Mailand, beim EASD-Kongress in Stockholm hingewiesen hat: Gibt man zu Metformin Vildagliptin dazu, sinkt nicht nur der $\mathrm{HbA}_{1 c}$, sondern auch die Inzidenz gastrointestinaler (GI) Nebenwirkungen. In einer aktuellen Studie traten mit bis zu 2 × 1 g Metformin am Tag bei 21\% der Patienten GI-Probleme auf, bei $2 \times 500 \mathrm{mg}$ plus jeweils $50 \mathrm{mg}$ Vildagliptin nur bei $15,4 \%$.

In einer Untersuchung von Bosi erhielten beide Gruppen dieselbe Metformindosis (Abb. 1). 2 x $1 \mathrm{~g} / \mathrm{d}$ als Monotherapie führten bei 11\% der Patienten zu Durchfall, kombinierte man die Standarddosis Vildagliptin dazu nur bei $6,5 \%$. Dies sei ein Hinweis darauf, dass das Gliptin die unerwünschten GI-Effekte von Metformin abschwächen könnte.

(spa) II

II Symposium und Meet the Expert, EASD-Kongress, Stockholm, 20. und 22. September 2010 (Veranstalter: Novartis)

\section{Nach Herzinfarkt und Koronarintervention Neuer Plättchenhemmer in den Leitlinien empfohlen}

In der neuen Leitlinie der Europäischen Gesellschaft für Kardiologie (ESC) zur myokardialen Revaskularisation wird für Patienten mit ST-Hebungsinfarkt (STEMI), die sich einer perkutanen Koronarintervention (PCI) unterziehen müssen, nun erstmals Prasugrel empfohlen - es sei denn, es bestehen Kontraindikationen. Die neue Empfehlung gilt insbesondere für Patienten mit primärer $\mathrm{PCl}$ und Stentimplantation. Prasugrel wird auch für die Langzeittherapie zuhause empfohlen, und zwar für zwölf Monate.
I. Bisher war man froh, mit Clopidogrel einen potenteren Hemmer der Antikoagulation als ASS in Händen zu haben. „Aber das Bessere ist der Feind des Guten“, so Prof. Sigmund Silber, München. In diesem Sinne bietet Prasugrel klare Vorteile gegenüber Clopidogrel, und zwar hinsichtlich zweier klinisch relevanter Aspekte: zum einen aufgrund der Verlässlichkeit der Wirkung. Prasugrel muss nämlich nicht aus einem Prodrug in das wirksame Arzneimittel umgewandelt werden, während die wirksame Fraktion von Clopidogrel je nach genetischem Background unterschiedlich groß ist. Zum anderen wegen der Unabhängigkeit der Wirkung von einer unter Umständen gleichzeitig erforderlichen Therapie mit Protonenpumpenhemmern. Die Wirkung von Clopidogrel wird dagegen abgeschwächt. Die Verlässlichkeit der dualen Plättchenhemmung ist aber essenziell, insbesondere um die oftmals tödlichen InStent-Thrombosen nach einer Stentimplantation zu verhindern.

(wk) II

II Media Briefing Meeting, ESC-Kongress, Stockholm, 31. August 2010 (Veranstalter: Daiichi-Sankyo / Lilly) 